
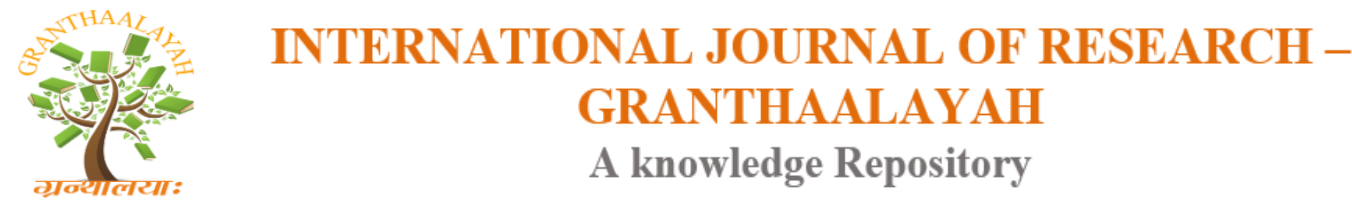

Science

\title{
KHADIRARISHTA: A MEDICAL REVIEW
}

\author{
Dr.Rashmi Rajendra Shinde ${ }^{1}$, Dr.Kirti Bhangale ${ }^{2}$ \\ ${ }^{1}$ M.D. 2ND year (Sch) (Agadtantra Vyavahar Ayurved Evam Vidhivaidyak) PDEA's College of \\ Ayurved and Research Centre, Nigdi, Pune 44, India \\ ${ }^{2}$ M.D. (Dravyaguna) Assistant Professor (Agadtantra) PDEA's College of Ayurved and \\ Research Centre, Nigdi, Pune 44, India
}

\begin{abstract}
In sushrut samhita chikitsa6/19 it has been mentioned that khadira is effective in all type of kushta. Khadirarishta is a very effective Arishta in the treatment of skin diseases specially Ekakushta. It contains 5-10\% of self-generated alcohol in it. It mainly contains Khadira which is potent krumighna and kandughna dravya.
\end{abstract}

Keywords: Khadirarishta; Khadira; Medical.

Cite This Article: Dr.Rashmi Rajendra Shinde, and Dr.Kirti Bhangale. (2017). "KHADIRARISHTA: A MEDICAL REVIEW." International Journal of Research Granthaalayah, 5(10), 72-75. https://doi.org/10.29121/granthaalayah.v5.i10.2017.2270.

\section{Introduction}

\section{Ingredients}

The contents of Khadirarishta are as follows

- Khadira - Acacia catechu, Devdaru- Cedrus deodara

- Bakuchi- Psoralea corylifolia, Daruharidra- Berberis aristata

- Haritaki- Terminalia chebula, Bibhitaka- Terminalia bellerica, Amalaki- Emblica officinalis

\section{Prakshepa Dravyas}

- Dhataki- woodfordia fruticosa, Kankola - Piper cubeba, Nagakeshar - Mesua ferrea

- Jatiphala - Myristica fragrance, Lavanga - Syzygium aromaticum

- Ela - Elettria cardmoum, Tvak \& Twakpatra - Cinamonum zeylonica.

- Pippali - Piper longum

\section{Other Dravyas}

- Madhu - Honey

- Sharkara - Crystallized sugar lumps, Water 
Characteristics of ingredients of Khadirarishta

\begin{tabular}{|c|c|c|c|c|c|}
\hline Dravya & Rasa & Vipaka & Virya & Doshaghnata & Rogaghnata \\
\hline Khadira & Tikta, kashaya & Katu & Sheeta & $\begin{array}{l}\text { Kapha pitta } \\
\text { shamaka }\end{array}$ & $\begin{array}{l}\text { Kushtaghna, } \\
\text { krumighna, kasaghna, } \\
\text { vranaropan }\end{array}$ \\
\hline Devdaru & $\begin{array}{l}\text { Tikta, katu, } \\
\text { kashaya }\end{array}$ & Katu & Ushna & $\begin{array}{l}\text { Kapha- vata } \\
\text { shamaka }\end{array}$ & $\begin{array}{l}\text { Kushtaghna, } \\
\text { krumighna, dushta } \\
\text { vrana shodhana }\end{array}$ \\
\hline Bakuchi & Katu, tikta & Katu & Ushna & $\begin{array}{l}\text { Vata-kapha } \\
\text { shamaka }\end{array}$ & $\begin{array}{l}\text { Krumighna, } \\
\text { kandughna,jwaraghna, } \\
\text { swedajanana }\end{array}$ \\
\hline Daruharidra & Kashaya, tikta & Katu & Ushna & $\begin{array}{l}\text { Pitta-kapha } \\
\text { shamaka }\end{array}$ & $\begin{array}{l}\text { Shotaghna, } \\
\text { vedanasthpan, } \\
\text { vranashodhan-ropan, } \\
\text { chakshushya }\end{array}$ \\
\hline Haritaki & $\begin{array}{l}\text { Pahncharasat } \\
\text { maka } \\
\text { Lavana varjya }\end{array}$ & Madhur & Ushna & Tridoshahara & $\begin{array}{l}\text { Hrudya, kushtaghna, } \\
\text { jwaraghna, twachya, } \\
\text { krumighna }\end{array}$ \\
\hline Bubhitaka & Kashaya & Madhur & Ushna & $\begin{array}{l}\text { Pitta-kaphas } \\
\text { hamaka }\end{array}$ & $\begin{array}{l}\text { Jwarahara, kasahara, } \\
\text { virechanopaga }\end{array}$ \\
\hline Amalaki & $\begin{array}{l}\text { Pancharasatm } \\
\text { aka } \\
\text { Lavana varjya }\end{array}$ & Madhur & Sheeta & Tridoshaghna & $\begin{array}{l}\text { Dahashamana, keshya, } \\
\text { twachya }\end{array}$ \\
\hline
\end{tabular}

\section{Medical Properties}

1) Primary

- Aam Pachaka

- Antiprurities

- Anthelmintic

- Anti allergic

- Digestive stimulants

- Anti microbial

2) Secondary

- Anti-gout

- Anti histaminic

- Anti inflammatory

- Anti oxidant

- Hepato protective

\section{Indications}

Mainly used in symptoms like Itching, Rashes and sensitive skin. 


\section{Primary Uses}

1. Acne/pimples

2. Atopic dermatitis (Eczema)

3. Intestinal worms

4. Psoriasis

5. Skin allergies

6. Swollen lymph nodes

7. Urticaria

\section{Other Uses}

Spleen enlargement, liver disorders, anemia (generally due to worm infestation), tumors and cysts under the skin, heart diseases, cough and breathing troubles.

\section{Dose}

10-20 $\mathrm{ml}$ with luke-warm water.

\section{Previous Work Done}

1) Khadira

\section{A. Pharmacological Study}

- It has antioxidant property(V.Gayatri Devi, Anitha John,R.Sreekala Devi,A. Prabhakaran)

- Khadira is Anti-bacterial agent (Kushtaghna). It has Anti mycotic, Anti diarrhoeal properties. (Rohit kumar.khatik, Anita Sharma 2014).

- It has anti-fungal activity against dermatophytes. ( thilaga Thendral, T.Laxmi 2017).

\section{B. Clinical Study}

- Acacia catechu is used in conjunctivitis, Haemoptysis, Pruritis, Leprosy, eukoderma, Skin diseases, Nausea, diarrhoea. ( Kinnari Dhruve, C.R. Harisha,P.K.Prajapata oct-dec 2011)

2) Deodaru- It has Anti inflammatory, Diuretics, Anti pyretic and anti leprotic properties.

3) Bakuchi- It is used as Anti microbial, Anti bacterial, Anti fungal, Anti inflammatory.

4) Daruharidra- It is used as Rejuvenating effect. Also it is Anti pyretic, Anti pruritic, Anti haemorrhoidal properties.

5) Haritaki- It is used as Anti oxidant, Anti microbial, Anti diabetic, Hepato-protective, Anti inflammatory, Anti arthritis, Gastro intestinal and motility wound healing action.

6) Bibhitaki- It has Anti oxidant, Anti microbial, Anti diarrheal, Anti cancer, Anti diabetic, anti hypertensive, Hepato protective Action. 
7) Amalaki- It has hepato toxic and Anti tumour properties. It is used chronic stress and Hypo lipidemia.

\section{Side Effects}

Common side effect like burning sensation or heart burn occurs when taken without water.

\section{References}

[1] Bhaishajya Ratnawali 54/365-370.

[2] Sharangadhara samhita madhyam khanda 10/60-65.

*Corresponding author.

E-mail address: gauravkumar.shaha@ gmail.com 